and Chemical Reactions," Paper presented at Third Combustion and Propulsion Colloquium, NATO-AGARD, Palermo, Sicily, March 17-21, 1958.

28 Lees, L., "Laminar Heat Transfer Over Blunt-Nosed Bodies at Hypersonic Flight Speeds," Jet Propulsion, vol. 26 no. 4, 1956, pp. 259-269.

29 Liepmann, H. W., and Bleviss, Z. O., "The Effects of Dissociation and Ionization on Compressible Couette Flow," Douglas Aircraft Co., Report SM-19831, 1956.

30 Lighthill, M. J., "Dynamies of a Dissociating Gas, I-Equilibrium Flow," Journal of Fluid Mechanics, vol. 2, part 1, 1957, pp. 1-32.

31 Linnett, J. W., and Marsden, D. G. H., "The Kinetics of the Recombination of Oxygen Atoms at a Glass Surface," and "The Recombination of Oxygen Atoms on Salt and Oxide Surfaces," Proceedings of the Royal Aeronautical Society, Series A, no. 1199, vol. 234, March 1956, pp. 489-515.

32 Logan, J. G., Jr., "Relaxation Phenomena in Hypersonic Aerodynamics," Paper presented at IAS 25th Annual Meeting, Jan. 28-31, 1957 (IAS Preprint 728).

33 Meixner, J., "Zur Theorie der Warmeleitfahigkeit Reagierender Fluider Mischungen," Z. Naturforsch, vol. 7a, 1952, pp. 553-557.

34 Metzdorf, H. J., "Flows in Partly Dissociated Gases," Journal of The Aeronautical Sciences, vol. 25, no. 3, 1958, p. 200.

35 Moore, L. L., "A Solution of the Laminar Boundary Layer Equations for a Compressible Fluid with Variable Properties, Including Dissociation," Journal of the Aeronautical Sciences, vol. no. 8, 1952, pp. 505-518.

36 Nernst, W., "Chemisches Gleichgewicht und Temperaturgefallen," Festschrift Ludwig Boltzmann Gewidmet, 1904, pp. 904-915.

37 Penner, S. S., "Chemical Reactions in Flow Systems," NATO-AGARD, Butterworths, London, 1955.

38 Penner, S. S., Harshbarger, F., and Vali, V., "An Introduction to the Use of the Shock Tube for the Determination of Physico-Chemical Parameters," Combustion Researches and Reviews, NATO-AGARD, Butterworths, London, 1957, pp. 134-172.

39 Prigogine, I., and Buess, R., Acad. Royale de Belgique, vol. 38 , series 5, 1952, pp. 711-851.
40 Rabinowicz, J., "Aerodynamic Studies in the Shock Tube," GALCIT Hypersonic Research Project, Memorandum 38, June 1957.

41 Romig, M. F., and Dore, F. J., "Solutions of the Compressible Laminar Boundary Layer Including the Case of a Dissociated Free Stream,' Convair, Report ZA-7-012, 1954.

42 Rose, P. H., Probstein, R. F., and Adams, M. C., "Turbulent Heat Transfer Through a Highly-Cooled, Partially Dissociated Boundary Layer,' Avco Research Laboratory, Research Report 14, Jan. 1958.

43 Rose, P. H., and Stark, W. I., "Stagnation Point Heat Transfer Measurements in Dissociated Air," Journal of the Aeronautical Sciences, vol. 25, no. 2, 1958, p. 86.

44 Rosner, D. E. "Boundary Conditions for the Flow of a Multi-Component Gas Near a Reactive Wall," Jet Propulsion (to appear).

45 Rosner, D. E., "Chemically Frozen Boundary Layers with Surface Reaction," Princeton University Aeronautical Engineering Report 419, March 1958; submitted to Journal of Aeronautical Sciences.

46 Scala, S. M., "Hypersonic Heat Transfer to Catalytic Surfaces," Journal of the Aeronautical Sciences, vol. 25, no. 4, 1958 , pp. 273-275.

47 Scala, S. M., "Hypersonic Stagnation Point Heat Transfer to Surfaces Having Finite Catalytic Efficiency," Paper presented at the Aerothermochemistry Session, Third U.S. National Congress of Applied Mechanics, Brown University, June 13, 1958.

48 Schotte, W., "Heat Transfer to a Gas Phase Chemical Reaction," Industrial Engineering and Chemistry, vol. 50, no. 4, 1958 , pp. $683-690$

49 Ubbelohde, A. R., Journal of Chemical Physics, vol. 3, 1915 , p. 69.

50 Ziebland, H., "Some Experimental Observations Regarding the Convective Heat Transfer from Highly Dissociated Combustion Gases to Cooled Walls of Rocket Engines," Ministry of Supply; Explosives Research and Development Establishment, Great Britain, Technical Memorandum 13/M/56, Aug. 1956.

51 Zigrang, D. J., "Note on Dissociation Effects in Hypersonic Viscous Flows," Journal of the Aeronautical Sciences, vol. 24 , no. 12,1957 , p. 916 .

\title{
A Preliminary Study of the Application of Steady- State Detonative Combustion to a Reaction Engine
}

\author{
R. DUNLAP, ${ }^{2}$ R. L. BREHM ${ }^{2}$ and J. A. NICHOLLS ${ }^{3}$
}

University of Michigan, Ann Arbor, Mich.

The purpose of this study is to determine the feasibility of a reaction engine employing a continuous detonation process at the combustion chamber. A reaction-type engine employing steady-state detonative combustion is considered. A simplified analysis treats the supersonic mixing of fuel and air together with the requirements necessary to achieve steady-state detonative combustion. Calculations of specific thrust and specific fuel consumption as functions of flight Mach number are made for hydrogen and acetylene fuels. The results of this study indicate that some supersonic diffusion of the air is necessary even though supersonic combustion exists. It is concluded that the speed range of air-breathing engines may be materially extended.

Received Nov. 13, 1957.

1 This research was supported by the United States Air Force through the Air Force Office of Scientific Research of the Air Research and Development Command, under Contract No. AF 18(600)-1199.

${ }^{2}$ Research Assistant, Engineering Research Institute.

${ }^{3}$ Instructor, Department of Aeronautical Engineering. Mem. ARS.

\section{Nomenclature}

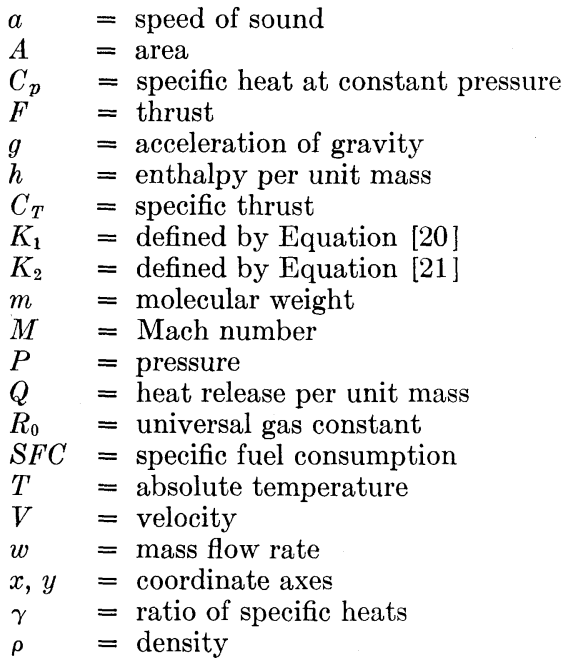




\section{Subscripts}

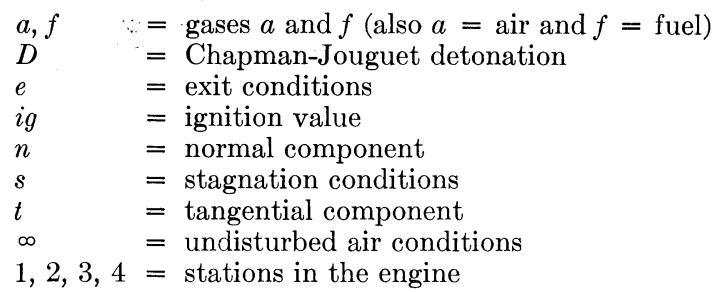

\section{Introduction}

$\mathbf{T}$ O DATE there has been considerable experimental and theoretical work devoted to the study of detonation waves. It has been found that upon ignition of various combustible gas mixtures under certain conditions of pressure, volume and composition, a flame front will propagate through the mixture at speeds ranging from 3000 to $12,000 \mathrm{fps}$. This front (shock-initiated combustion) is called a detonation wave and the speed at which it propagates is the detonation velocity. So far this phenomenon has been observed mainly in shock tubes in which the wave is in a transient state. At present, however, attempts are being made to accelerate a combustible gas at high total temperature and pressure to the local detonation velocity and then cause it to ignite, thus establishing a standing (steady-state) detonation wave (1). ${ }^{4}$

One is led to wonder if such a burning process could be applied to a propulsive system. It seems that a jet device utilizing this mode of combustion, as opposed to deflagration burning, would offer several advantages. For example, the supersonic inlet diffuser could be greatly simplified since the burning would occur at supersonic speeds. Thus the incoming air need not be diffused subsonically and hence shockswallowing problems, as well as large total pressure losses, could be eliminated. The static pressure rise, necessary for expelling the exhaust gases, would now be realized, at least in part, across the detonation wave rather than entirely through the diffuser. Furthermore, since the detonation process occurs at high velocities and total temperatures, it seems to be a natural means of extending the speed range of air-breathing vehicles. Other advantages would include a shortened combustion chamber with no need for an ignition device. An obvious disadvantage is the lack of static thrust.

In view of possible applications, a preliminary analysis was made in an effort to determine some of the performance characteristics of an engine in which heat is added by means of a standing detonation. Special attention is given to the supersonic mixing of fuel and air, and solutions are presented for the two cases when either the pressure or the area remain constant throughout the mixing zone. A general discussion of the detonation process, together with the method in which it could be applied to a steady-flow engine, is also given. Finally, problems associated with the matching of the various flow processes to produce an efficient thrust-producing mechanism are discussed and some over-all characteristics, such as specific thrust and specific fuel consumption, are calculated.

It should be pointed out that the analysis contained herein is limited to the following general assumptions: The working fluid is assumed to be an inviscid perfect gas mixture. Average values of the specific heats, applicable to the temperature range and mixture considered, are used throughout. Heat and frictional losses are assumed absent, and any total pressure losses due to the formation of oblique shock waves while compressing a supersonic gas are neglected. Finally, it is supposed that there exists an "effective ignition temperature" below which the fuel-air mixture must be kept prior to detonating.

${ }^{4}$ Numbers in parentheses indicate References at end of papers.

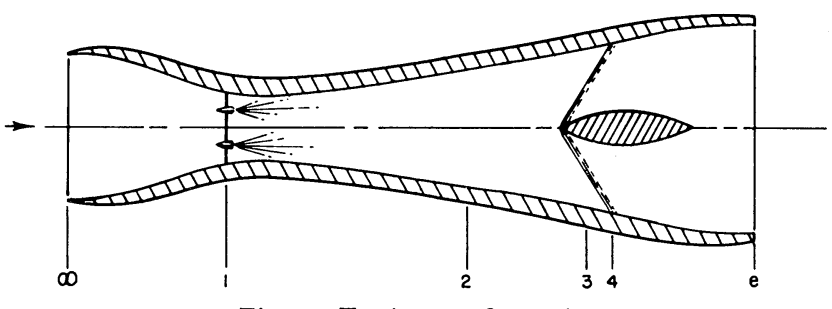

Fig. 1 Engine configuration

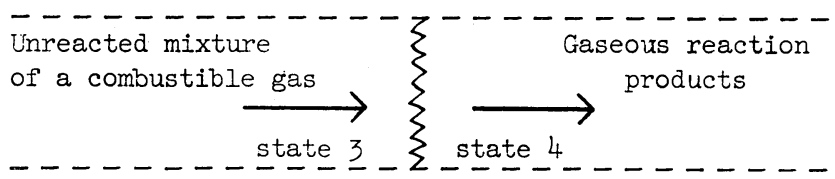

Fig. 2 Normal detonation wave

In view of these assumptions, it should be clear that the results presented in this paper can only indicate approximate values of performance characteristics comparable to other idealized engines, which is all that was intended.

\section{General Considerations}

The following is a brief discussion of the flow system assumed in the analysis of this engine. Fig. 1 is a sketch of this flow system.

The desirability of an engine requiring no diffusion of the incoming air has already been mentioned. At first one might think that no diffusion would be required in this engine since both mixing and combustion are occurring at supersonic velocities. However, it will be shown that for maximum pressure recovery through the engine there must be supersonic diffusion to some extent previous to mixing the fuel and air. This diffusion occurs between stations $\infty$ and 1.

The fuel is injected into the supersonic stream at station 1 and is assumed fully mixed with the air at station 2 .

Detonation occurs at station 3. Under certain conditions, namely, at low flight speeds, the back pressure should be sufficient to initiate the detonation normal to the flow at some station 3. However, this type of initiation may possibly be unstable, and in practice some form of stabilization, such as a thin body placed in the stream, will probably be required. At high flight speeds, certain mixing requirements and efficiency considerations dictate that a body must definitely be placed in the stream. The resulting oblique shock wave initiates the chemical reaction and the body serves as the means of stabilization. For the oblique wave portion of this analysis, the detonation is assumed to be stabilized on a twodimensional wedge.

Chemical equilibrium conditions are assumed to exist at station 4 immediately downstream of the detonation. Finally, frozen equilibrium flow is assumed in the isentropic expansion to atmospheric pressure at the exit. Downstream reflections of the stabilized wave are neglected and of course variable geometry is implied.

It should be pointed out that the question of stability of the assumed mode of combustion is as yet an unresolved one. In fact, this is the primary objective of the Air Force contract supporting the study reported here. This problem is being attacked both theoretically and experimentally. For purposes of this paper, stability has been implicitly assumed. It is felt that this is highly probable for wedge stabilization although it is admitted that a normal detonation wave existing between stations 2 and 3 would in all likelihood be unstable and some means of stabilization would be required. Such a wave could not move all the way upstream to position 1 , however, due to incomplete mixing.

In the over-all consideration of the analysis, two areas of 
special interest and importance stand out-the supersonic mixing of fuel and air and the detonation process. Each will be considered separately in the following two sections.

\section{Considerations of the Detonative Process}

For purposes of analysis a detonation wave is usually treated as a discontinuity with heat addition. ${ }^{5}$ First, consider the one-dimensional model of a detonation in which the reference system is attached to the wave and the gases move relative to it (Fig. 2).

The conservation equations for this system can be written

$$
\begin{aligned}
& \text { mass: } \quad \rho_{3} V_{3}=\rho_{4} V_{4} \quad \text { or } \quad \frac{\gamma_{3} M_{3} P_{3}}{a_{3}}=\frac{\gamma_{4} M_{4} P_{4}}{a_{4}} \ldots[1] \\
& \text { momentum: } P_{3}+\rho_{3} V_{3}{ }^{2}=P_{4}+\rho_{4} V_{4}{ }^{2} \quad \text { or } \\
& P_{3}\left(1+\gamma_{3} M_{3}{ }^{2}\right)=P_{4}\left(1+\gamma_{4} M_{4}{ }^{2}\right) \ldots[2] \\
& \text { energy: } C_{p_{3}} T_{3}+\frac{V_{3}{ }^{2}}{2}+Q=C_{p_{4}} T_{4}+\frac{V_{4}{ }^{2}}{2} \text { or } \\
& \frac{a_{3}{ }^{2}}{\gamma_{3}-1}\left(1+\frac{\gamma_{3}-1}{2} M_{3}{ }^{2}\right)+Q=\frac{a_{4}{ }^{2}}{\gamma_{4}-1}\left(1+\frac{\gamma_{4}-1}{2} M_{4}{ }^{2}\right)
\end{aligned}
$$

Next, consider an extension of the above model to the twodimensional case wherein an oblique detonation is stabilized on a wedge (Fig. 3). In this model the incoming velocity is resolved into components normal and tangential to the wave. Since there is no net pressure force in the tangential direction, there can be no change in the tangential velocity across the wave. The normal components may be treated as in the onedimensional case. Hence the two-dimensional analysis reduces to the vector addition of a (tangential) velocity to the one-dimensional normal wave model. Note that the conservation equations for this model are identical to the previous one-dimensional equations except that $V_{3}$ and $V_{4}$ are now understood to be the normal components of the total velocities. Note also that in the energy equation the tangential components of the velocity will cancel.

One finds upon analyzing the above conservation equations that, for a given upstream temperature and heat release, solutions to the equations exist only for upstream velocities above a certain minimum. For all velocities above this minimum, the equations predict two possible solutions for the downstream conditions; however, one solution is ruled out by entropy considerations. ${ }^{6}$

It is interesting to note that the minimum velocity is the velocity always measured in shock-tube investigations of detonation. Associated with this minimum velocity are downstream conditions such that the Mach number of the burned gases relative to the wave is always unity. Also, the total pressure loss across such a detonation is a minimum. This type of wave is called a Chapman-Jouguet detonation.

Since total pressure is of prime importance in the efficient conversion of thermal energy into kinetic energy, the total pressure loss across the detonation in this engine was minimized by imposing the conditions necessary for a ChapmanJouguet type wave. This proves to be a fortunate choice from still another standpoint since the heat release computation can be eliminated. Thus, by using experimental values for the detonation velocity, the momentum and mass equations can be solved without considering the energy equation, and hence the laborious chemical equilibrium calculations behind the wave are avoided.

\section{Mixing Analysis}

An exact solution to the supersonic mixing of fuel and air

${ }^{5}$ It may be thought of as a shock wave with combustion.

${ }^{6}$ See (1) for a comprehensive discussion of the oblique wave solution.

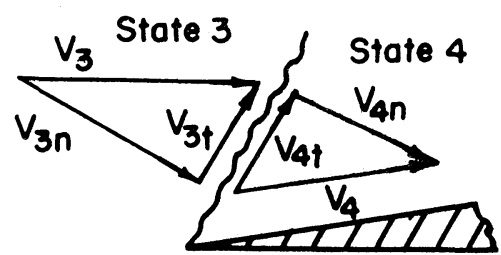

Fig. 3 Oblique detonation wave

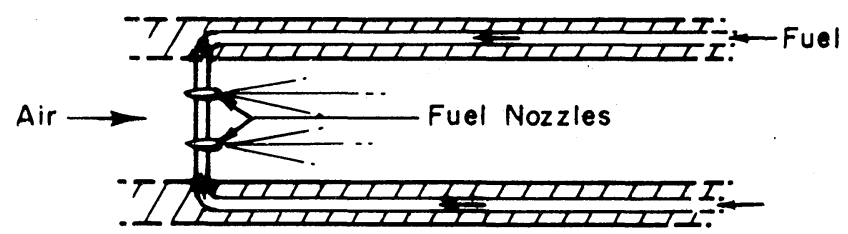

Fig. 4 A method of fuel injection

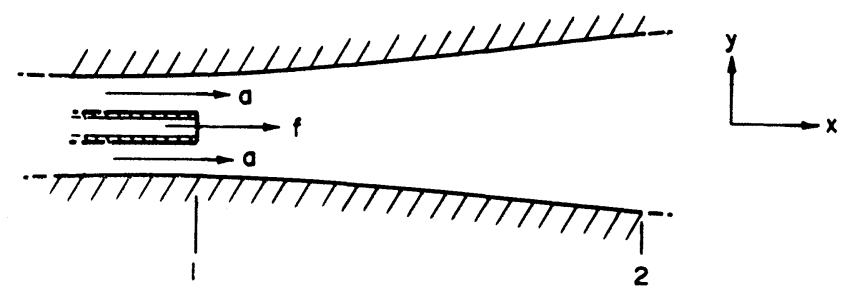

Fig. 5 Mixing region

would be prohibitively complicated. Consider, for example, a scheme for introducing gaseous fuel into a supersonic stream of air as shown in Fig. 4. Air, at a high stagnation temperature and pressure, would flow around the nozzles at supersonic speeds and proceed to mix with the fuel through molecular and turbulent diffusion. The resulting shear flow would be nonuniform and nonsteady. The flow would be further complicated by the presence of shock waves generated in the nozzle region, as well as by possible local burning in the boundary layer in the vicinity of the nozzle exit. Since it would clearly be quite difficult to make a detailed investigation, the following analysis will be directed toward a simplified solution of the mixing problem.

Consider the mixing of two inviscid perfect gases (denoted by subscripts $a$ and $f$ ) as shown in Fig. 5 . It is assumed that the rate of change of any flow parameter in the $x$ or $y$ direction is zero at stations 1 and 2 (except at the initial interface of the gases where a discontinuity may exist in the $y$ direction in the temperature and velocity), and that the gases are fully mixed at station 2 .

With these assumptions the conservation equations may be written in difference form as

$$
\begin{aligned}
\text { mass: } \quad & \rho_{1 a} V_{1 a} A_{1 a}+\rho_{1 j} V_{1 j} A_{1 j}=\rho_{2} V_{2} A_{2} \ldots \ldots \ldots \ldots . \\
\text { energy: } \quad & w_{1 a}\left(h_{1 a}+\frac{V_{1 a}{ }^{2}}{2}\right)+w_{1 f}\left(h_{1 j}+\frac{V_{1 f^{2}}}{2}\right)= \\
& w_{2}\left(h_{2}+\frac{V_{2}{ }^{2}}{2}\right) .
\end{aligned}
$$

momentum in $x$-direction:

$$
\begin{aligned}
& P_{1}\left(A_{1 a}+A_{1 j}\right)-P_{2} A_{2}+\int_{\rho_{2} A_{2} V_{2}{ }^{2}-\rho_{1 a} A_{1 a} V_{1 a}{ }^{2}-\rho_{1 j} A_{1 j} V_{1 j}{ }^{2} .} P d A=
\end{aligned}
$$

Using the equation of state

$$
P=\rho \frac{R_{0}}{m} T
$$

and approximating the enthalpy by

$$
h=C_{p} T
$$


where $C_{p}$ is an average value of the specific heat between $T=$ 0 and $T=T_{1}$, the conservation equations are rewritten in the form

mass:

$$
\frac{\gamma_{1 a} M_{1 a} A_{1 a} P_{1}}{a_{1 a}}+\frac{\gamma_{1 f} M_{1 s} A_{1 f} P_{1}}{a_{1 f}}=\frac{\gamma_{2} M_{2} A_{2} P_{2}}{a_{2}}
$$

energy: $\quad T_{s 2}=T_{2}\left(1+\frac{\gamma_{2^{-1}}}{2} M_{2}^{2}\right)=$

$$
\left\{\frac{\left[\frac{C_{p_{1 a} a}}{C_{p_{1 f}}} \frac{w_{1 a}}{w_{1 f}}+\frac{T_{s_{1 f}}}{T_{s_{1} a}}\right]}{\left[\frac{C_{p_{1 a}}}{C_{\rho_{1 f}}} \frac{w_{1 a}}{w_{1 f}}+1\right]}\right\} T_{s_{1 a}} .
$$

momentum: $\quad P_{1} A_{1}\left(1+\gamma_{1 a} \frac{A_{1 a}}{A_{1}} M_{1 a^{2}}+\gamma_{1 f} \frac{A_{1 f}}{A_{1}} M_{1 f^{2}}\right)+$

$$
\int_{A_{1}}^{A_{2}} P d A=P_{2} A_{2}\left(1+\gamma_{2} M_{2}{ }^{2}\right)
$$

where

$$
A_{1}=A_{1 a}+A_{1 f}
$$

Notice that the momentum equation contains an integral term that is dependent upon the variation of pressure during the mixing, while the other conservation equations express a relationship between the end points only. It is apparent that a solution can be found for constant pressure mixing, in which case

$$
\int_{A_{1}}^{A_{2}} P d A=P_{1}\left(A_{2}-A_{1}\right)=P_{2}\left(A_{2}-A_{1}\right) .
$$

or for constant area mixing where

$$
\int_{A_{1}}^{A_{2}} P d A=0 \text {. }
$$

In general the integral can be approximated by using an average constant value of the pressure during mixing although this case will not be considered.

By combining Equations [7, 8, 9] and [10 or 11], the following solutions for the downstream Mach number are found for constant pressure or constant area mixing

constant pressure mixing: $\phi\left(M_{2}\right)=$

$$
\frac{\left[\frac{\gamma_{1 a}}{\gamma_{2}} \frac{m_{2}}{m_{1 a}}\left(\frac{1+\frac{C_{p_{1 a} a}}{C_{p_{1 j}}} \frac{w_{1 a}}{w_{1 j}}}{\frac{T_{s_{1} f}}{T_{s_{1} a}}+\frac{C_{p_{1 a}}}{C_{p_{1 j}}} \frac{w_{1 a}}{w_{1 j}}}\right)\right]^{1 / 2}\left[\phi\left(M_{1 a}\right)+\frac{a_{s_{1 j}}}{a_{s_{1 a}}} \frac{w_{1 f}}{w_{1 a}} \phi\left(M_{1 f}\right)\right]}{\left[1+\frac{w_{1 f}}{w_{1 a}}\right]}
$$

where

$$
\phi(M)=\frac{M}{\sqrt{1+\frac{\gamma-1}{2} M^{2}}}
$$

constant area mixing: $\eta\left(M_{2}\right)=$

$$
\frac{\left[\frac{\gamma_{1 a}}{\gamma_{2}} \frac{m_{1 a}}{m_{2}}\left(\frac{\frac{T_{s_{1}}}{T_{s_{1 a}}}+\frac{C_{p_{1 a} a}}{C_{p_{1} f}} \frac{w_{1 a}}{w_{1 f}}}{1+\frac{C_{p_{1 a} a}}{C_{p_{1 j}}} \frac{w_{1 a}}{w_{1 f}}}\right)\right]^{1 / 2}\left[1+\frac{w_{1 j}}{w_{1 a}}\right]}{1+\frac{\gamma_{1 a}}{\gamma_{1 j}} \frac{w_{1 f}}{w_{1 a}} \frac{a_{s_{1} f}}{w_{s_{1} a}} \frac{\eta\left(M_{1 a}\right)}{\eta\left(M_{1 f}\right)}}
$$

where

$$
\eta(M)=\frac{M \sqrt{1+\frac{\gamma-1}{2} M^{2}}}{\left(1+\gamma M^{2}\right)}
$$

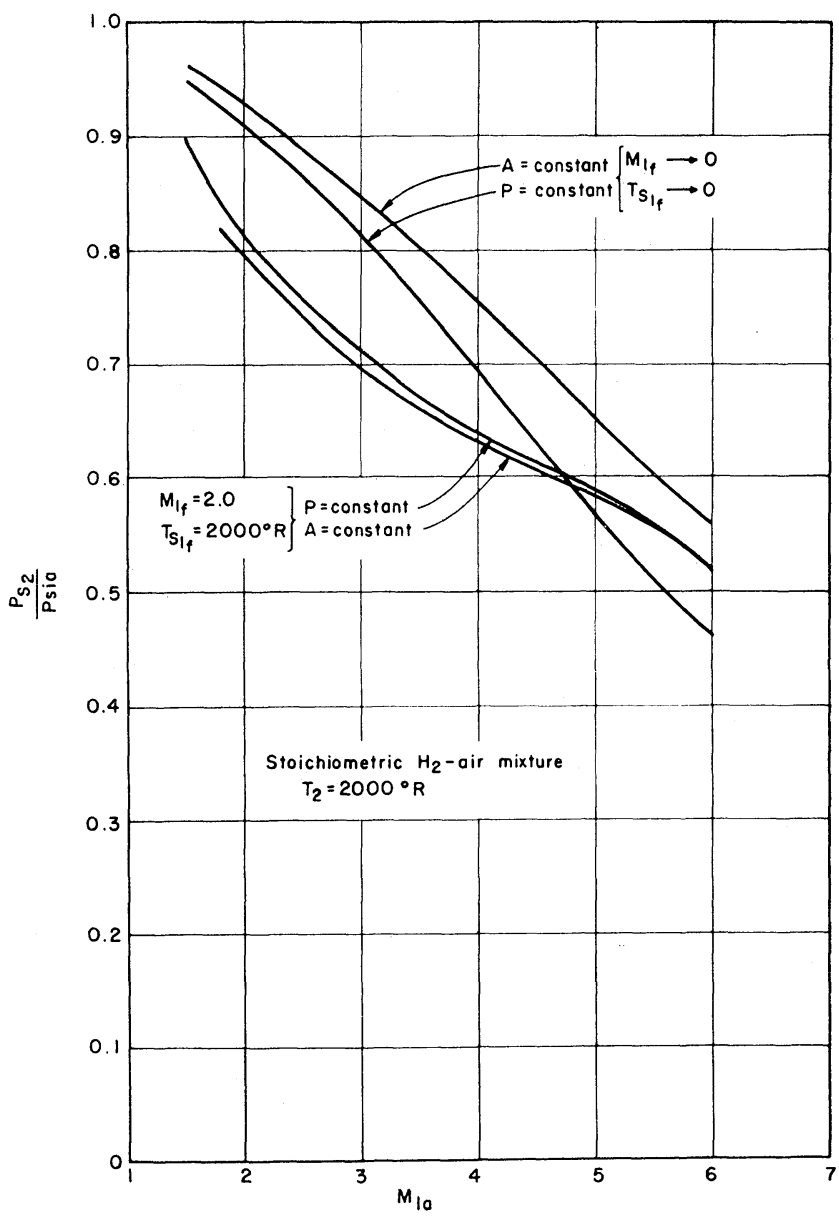

Fig. 6 Total pressure ratio across the mixing zone

It should be understood that the subscript for $\gamma$ in $\eta(M)$ and $\phi(M)$ should correspond to the subscript for $M$.

Of particular interest in the engine analysis are the downstream total temperature, given directly by Equation [8], and the downstream total pressure. The total pressure ratios across the mixing zone for the two cases are given for $P_{1}=P_{2}$ by

$$
\frac{P_{s 2}}{P_{s_{1 a}}}=\frac{\left[1+\frac{\gamma_{2-1}}{2} M_{2}^{2}\right] \frac{\gamma_{2}}{\gamma_{2-1}}}{\left[1+\frac{\gamma_{1 a-1}}{2} M_{1 a^{2}}\right] \frac{\gamma_{1 a}}{\gamma_{1 a-1}}} .
$$

and for $A_{1}=A_{2}$

$$
\begin{gathered}
\frac{P_{s 2}}{P_{s_{1} a}}=\frac{\left[1+\frac{\gamma_{2-1}}{2} M_{2}^{2}\right] \frac{\gamma_{2}}{\gamma_{2-1}}}{\left[1+\frac{\gamma_{1 a-1}}{2} M_{1 a^{2}}\right]^{2} \frac{\gamma_{1 a}}{\gamma_{1 a-1}}} \times \\
\frac{\left(1+\gamma_{1 a} \frac{A_{1 a}}{A_{1}} M_{1 a^{2}}+\gamma_{1 f} \frac{A_{1 f}}{A_{1}} M_{1 f}{ }^{2}\right)}{\left(1+\gamma_{2} M_{2}^{2}\right)} .
\end{gathered}
$$

Fig. 6 shows a plot of the total pressure ratio as a function of $M$ for a stoichiometric hydrogen-air mixture. For this case, and in the remainder of the report, the subscripts $a$ and $f$ will refer to the air and fuel, respectively. The downstream boundary condition $\left(T_{2}=2000 \mathrm{R}\right)$ was chosen so that the calculations would correspond to a practical case wherein the combustible mixture must be kept below an effective ignition temperature. Curves for two different fuel-inlet conditions are shown. It can be seen that as the Mach number of the air increases the total pressure ratio continually decreases 
and that in the Mach number range between $M=2$ and $M=$ 4 the least loss in total pressure is obtained by injecting the fuel at the low energy level. It is physically plausible that there should be a better total pressure recovery (referred to the air total pressure) when the fuel is at a low energy level since more thermal energy from the air must be transferred to the fuel. ${ }^{7}$ As the Mach number of the air increases, the air total temperature becomes so large that the effect of the difference in fuel-inlet conditions becomes less significant.

The results shown in Fig. 6 indicate the desirability of diffusing the incoming air somewhat before injecting the fuel because of the importance of total pressure recovery in engine performance.

\section{Detailed Examination of the Flow System}

The integration of the previous work into an over-all engine system will now be considered.

An important limitation peculiar to this type of engine is that the static temperature of the unreacted fuel-air mixture must be kept below an "effective ignition temperature." The "effective ignition temperature" is herein defined as that temperature at which a moving combustible gas mixture will spontaneously ignite. Clearly, this temperature will depend on the dynamic and thermodynamic state of the gas, composition, confining boundaries, the flow process involved, etc. To the authors' knowledge, no studies involving all these parameters have been made. To effect the calculations for this engine, it was necessary to assume some value of ignition temperature which appeared reasonable on the basis of known values found in the literature for stagnant mixtures.

In applying the ignition-temperature condition, certain ramifications must be considered. The total pressure loss across the detonation can be minimized by the attainment of a Chapman-Jouguet detonation at the lowest possible detonation Mach number (3). Since this Mach number varies inversely as the square root of the temperature, ${ }^{8}$ it is obvious that the detonation should occur at the highest possible temperature. This is advantageous from still another standpoint since, as can be seen from Fig. 6, the total pressure losses through the mixing zone are decreased when the Mach number of mixing is decreased. Hence, for best performance, the static temperature of the unreacted gas must be allowed to approach its limiting value, the ignition temperature. Also, from this reasoning, it is concluded that supersonic diffusion of the air prior to mixing with the fuel is necessary.

In locating the detonation in this engine, the above discussion would indicate that it should coincide with the point of maximum static temperature, namely, at the end of mixing. Recall, however, that to achieve detonation, a certain minimum velocity is required. At low flight speeds this minimum velocity will not be realized at the end of mixing, and the flow must be expanded. The detonation will then occur at some downstream position (station 3, Fig. 1), as a Chapman-Jouguet normal wave. As the flight speed is increased, the conditions sufficient to generate this type of detonation will occur further upstream until finally they will exist just at the end of mixing. For any higher flight speeds, the wave must be stabilized on a wedge so that the normal component of velocity remains a minimum (Chapman-Jouguet oblique wave).

With the general engine configuration thus determined, it is possible to compute over-all performance. By specifying the flight Mach number, ambient air temperature, fuel, fuel-air ratio and fuel-inlet conditions, the computations are made as follows:

${ }^{7}$ Recall that in a frictionless heat subtraction process the total pressure of the gas will increase.

${ }^{8}$ The Chapman-Jouguet detonation velocities used in this report were assumed to be independent of initial temperature and pressure and were obtained from (2).

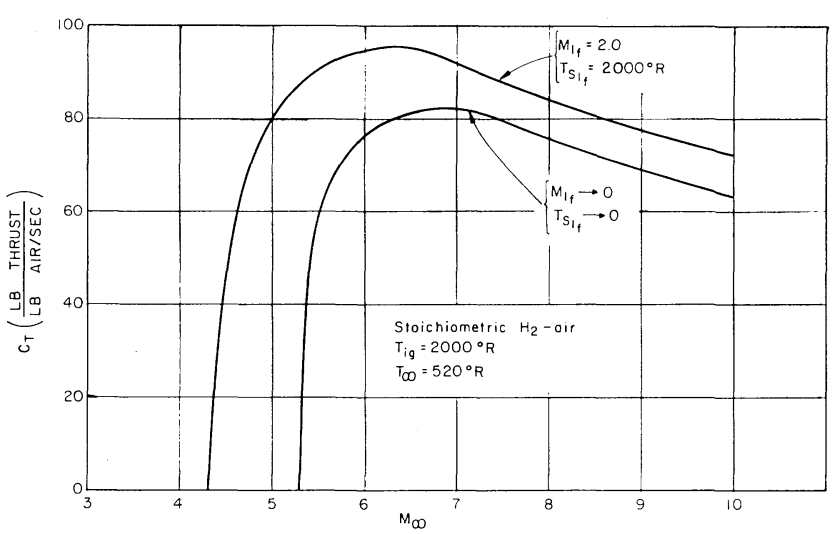

Fig. 7 Specific thrust vs. flight Mach number

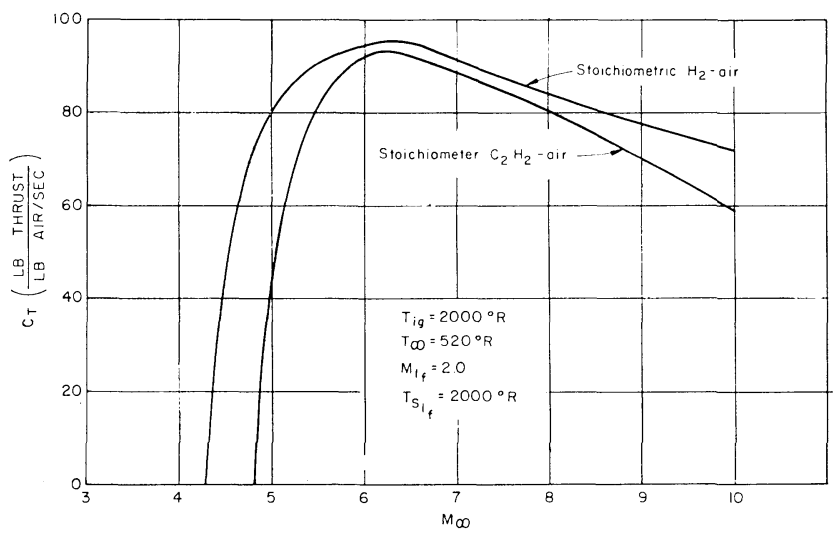

Fig. 8 Specific thrust vs. flight Mach number

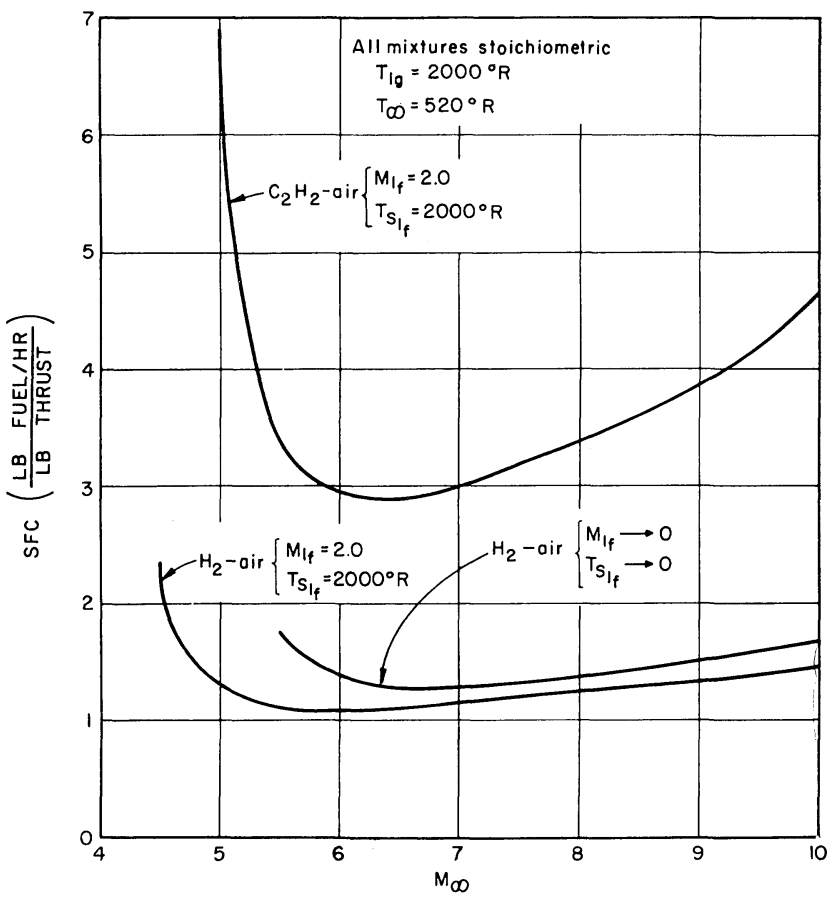

Fig. 9 Specific fuel consumption vs. flight Mach number 
(a) Setting $T_{2}=T_{i g}$, determine $M_{2}$ and $T_{s 2}$ from Equation [8].

(b) Knowing $M_{2}$ and $T_{s 2}$, compute $M_{1 a}$ from Equation [12 or 14].

(c) Calculate $P_{s 2} / P_{s 1 a}$ from Equation [16 or 17].

(d) Find $V_{2}=M_{2} a_{2}$ and compare with $V_{D}$.

1. If $V_{2}$ is less than $V_{D}$, use the normal wave solution below (Equation [18]) to compute $V_{e}$.

2. If $V_{2}$ is greater than $V_{D}$, use the oblique wave solution (Equation [19]).

The following equations were derived by use of the conservation equations between the end of mixing and the exit. The solution across the detonation wave was determined so that a Chapman-Jouguet (normal or oblique) wave always occurred.

normal detonation:

$$
\begin{aligned}
& V_{e}=\frac{\frac{\gamma_{4}}{\gamma_{2}}\left[a_{s 2}{ }^{2}+\frac{\gamma_{2+1}}{2} V_{D^{2}}\right]}{V_{D} \sqrt{\overline{\gamma^{2}{ }_{4-1}}}} \times \\
& \left\{1-\frac{\frac{2}{\left(\gamma_{4+1}\right)^{1 / \gamma_{4}}}\left(\frac{P_{\infty}}{P_{s 2}}\right)^{\frac{\gamma_{4-1}}{\gamma_{4}}}}{\left[1+\frac{\gamma_{2+1}}{2} \frac{V_{D}{ }^{2}}{a_{s 2}{ }^{2}}\right]^{\frac{\gamma_{4}-1}{\gamma_{4}}}\left[1-\frac{\gamma_{2-1}}{2} \frac{V_{D}{ }^{2}}{a_{s 2}{ }^{2}}\right]^{\frac{\gamma_{4}-1}{\gamma_{4}\left(\gamma_{2-1}\right)}}}\right\}^{1 / 2} .
\end{aligned}
$$

oblique detonation:

$$
\begin{aligned}
V_{e}=\left\{K_{1}+V_{2}{ }^{2}-K_{2}\left(\frac{P_{\infty}}{P_{s 2}}\right)^{\frac{\gamma_{4}-1}{\gamma_{4}}}\right. & \times \\
& \left.\left(a_{2}{ }^{2}+\frac{\gamma_{2-1}}{2} V_{2}{ }^{2}\right) \frac{\gamma_{2}\left(\gamma_{4-1}\right)}{\gamma_{4}\left(\gamma_{2-1}\right)}\right\}^{1 / 2} \ldots
\end{aligned}
$$

where

$$
\begin{gathered}
K_{1}=\left(\frac{\gamma_{4}}{\gamma_{2}}\right)^{2} \frac{\left(a_{2}{ }^{2}+\gamma_{2} V_{\left.D^{2}\right)^{2}}\right.}{\left(\gamma^{2}{ }_{4-1}\right) V_{D^{2}}}-V_{D}{ }^{2} \ldots \ldots \ldots \\
K_{2}=\left(\frac{\gamma_{4}}{\gamma_{2}}\right)^{2} \frac{2}{V_{D^{2}\left(\gamma_{4^{-1}}\right)}}\left(\frac{a_{2}{ }^{2}+\gamma_{2} V_{D}{ }^{2}}{1+\gamma_{4}}\right)^{\frac{\gamma_{4+1}}{\gamma_{4}}}\left(\frac{1}{a_{2}{ }^{2}}\right) \frac{\gamma_{4-1}}{\gamma_{4}\left(\gamma_{2-1}\right)} \ldots
\end{gathered}
$$

Performance characteristics such as specific impulse and specific fuel consumption can now be computed by the usual formulas

$$
\begin{gathered}
C_{T}=\frac{F}{w_{1 a}}=\frac{V_{e}\left(1+\frac{w_{1 f}}{w_{1 a}}\right)-V_{\infty}}{g}, \sec \ldots \ldots \\
S F C=3600 \frac{w_{1 f} / w_{1 a}}{C_{T}}, H R^{-1} \ldots \ldots \ldots \ldots
\end{gathered}
$$

\section{Results and Conclusions}

Figs. 7, 8 and 9 show the results of calculations of specific thrust and specific fuel consumption for hydrogen and acetylene fuels. In each case the fuel-air mixture was stoichiomet- ric and the effective ignition temperature was assumed to be $2000 \mathrm{R}$. The Chapman-Jouguet detonation velocities were approximately equal at a value of $5900 \mathrm{fps}$. The constant pressure mixing solution was used throughout.

From these graphs, it can be seen that there is no thrust below a flight Mach number of 4 and that the specific thrust reaches a maximum between Mach 6 and 7 , then decreases. The shape of the specific thrust curve can be explained as follows: First, below a certain flight speed the total energy of the fuel-air mixture is insufficient to achieve steady detonation. At slightly higher flight speeds a detonation will occur if the gases are expanded to a very high Mach number. However, for very high detonation Mach numbers, the total pressure loss is excessive and no thrust is realized. As the flight speed is increased, the Mach number of detonation decreases, and hence the specific thrust begins to increase. Finally the specific thrust reaches a maximum when the detonation is stabilized at its limiting position (the end of mixing). For still higher flight speeds the Chapman-Jouguet oblique wave solution exists at a constant Mach number of detonation at $T_{i g}$, and hence the total pressure ratio across the wave remains constant. Thus the specific thrust decreases because of the rising total pressure loss associated with the mixing process.

The importance of fuel inlet conditions can be seen from Fig. 7. The upper curve corresponds to $M_{1 f}=2$ and $T_{s i f}=$ $2000 \mathrm{R}$, while the other curve represents the lower limiting values for these parameters. The fuel-inlet conditions are seen to be quite important in determining the operating range for this type of engine.

The curves in Figs. 8 and 9 compare two different fuels. Since the detonation velocities were the same for both mixtures and the effective ignition temperatures assumed equal, the differences in these curves are due to the unequal molecular weights, fuel-air ratios, and specific heat ratios.

In deciding upon the feasibility of the proposed engine, the specific thrust and specific fuel consumption may be compared to an ideal ramjet. The present engine offers comparable performance at much higher flight Mach numbers, and indicates a possible means of extending the speed range of air-breathing vehicles. Finally, it must be stressed that these performance calculations are highly dependent on the effective ignition temperature assumed and are thus limited by the accuracy of this assumption. Furthermore, the results have not been optimized from the standpoint of mixing process and fuel-inlet conditions.

\section{References}

1 Rutkowski, J., and Nicholls, J. A., "Considerations for the Attainment of a Standing Detonation Wave," Proceedings of the Gas Dynamics Symposium, Northwestern University, 1956. Also issued as OSR-TN-55-216.

2 Lewis, B., and Von Elbe, G., "Combustion, Flames and Explosions of Gases," Academic Press, New York, 1951.

3 Morrison, R. B., "A Shock Tube Investigation of Detonative Combustion," University of Michigan, Engineering Research Institute Report UMM-97, Ann Arbor, 1952. 